\title{
Combined Influence of Cellular and Mechanical Cues on Ex Vivo Expansion of Hematopoietic Stem/Progenitor Cells
}

\author{
Yun Gyeong Kang ${ }^{1}$, Eun Jin Lee ${ }^{1}$, Ji Eun Kim ${ }^{1}$, Yanru Wu² ${ }^{2}$ Jung-Woog Shin ${ }^{1,2,3}$ \\ ${ }^{1}$ Department of Biomedical Engineering, Inje University \\ Gimhae, Korea \\ kyg06@bse.inje.ac.kr; lej13@bse.inje.ac.kr; sjw@inje.ac.kr; kje@bse.inje.ac.kr \\ ${ }^{2}$ Department of Health Science and Technology, Inje University \\ Gimhae, Korea \\ wyr08@bse.inje.ac.kr; sjw@inje.ac.kr \\ ${ }^{3} \mathrm{CMDC} /$ Institute of Aged Life Redesign/UHARC, Inje University \\ Gimhae, Korea \\ sjw@inje.ac.kr
}

\section{Extended Abstract}

Hematopoietic stem/progenitor cell (HSPC) transplantation is used extensively in the treatment of several blood-related malignant and inherited diseases [1]. Ex vivo expansion of HSPCs has been investigated to improve the clinical outcome of HSPC transplantation, particularly in cases with limited grafting size. Ex vivo expansion of HSPCs is problematic, because proliferating cells tend to differentiate easily, presumably due to the lack of appropriate cues normally provided by the in vivo microenvironment. In nature, HSPCs are located mainly in the bone marrow interact with a specific microenvironment referred to as stem cell niche, which regulates HSPC fate [2]. Here, we co-cultured HSPCs with mesenchymal stem cells (MSCs) and divided the HSPCs into two fractions according to whether they came into adherent to MSCs or not. Additionally, we used hydrostatic pressure (HP) to mimic the physical conditions in vivo.

The expanded total nucleated cell (TNC) count for non-adherent cells was significantly higher than that of adherent cells. However, the adherent cells maintained the HSPC phenotype $\left(\mathrm{CD} 34^{+}\right.$and $\left.\mathrm{CD} 34^{+} \mathrm{CD} 38^{-}\right)$to a greater degree than the non-adherent cells over the course of 4 days. The adherent cells also had superior clonogenic potential as assessed by colonyforming cell (CFC) and long-term culture-initiating cell (LTC-IC) assays.

We also used a bioreactor to provide HP to reproduce the physical conditions of the HSPC niche. We found that expanded cells from groups subjected to HP tended to have higher numbers of TNCs than did those from groups that were not subjected to HP. Interestingly, when HP was applied, the HSPC phenotype frequency and clonogenic potential were significantly higher than when HP was not applied. However, when HSPCs were cultured without MSCs, HP application had little effect on the maintenance of the HSPC phenotype or the clonogenic potential of expanded cells. Therefore, HP and MSC co-culture had synergistic effects.

To identify the cause of these synergistic effects, we harvested co-cultured MSCs under different culture conditions and assessed gene expression for HSPC niche markers (ANGPT1, ANGPT2, JAG1, OPN and RUNX2) that are essential for HSPC survival in vivo. As expected, we found that the expression levels of all markers were significantly higher in MSCs co-cultured with HSPCs (H_M and H_M_HP) compared with those with MSC alone (MSC w/o HSPC).

Our study demonstrated that the direct contact between HPSCs and MSCs enhances the maintenance of an immature HSPC phenotype and function. Additionally, we showed that HP application significantly influences the outcome of HSPC expansion and the maintenance of stemness. It is the first study which applies HP to the expansion of HSPCs. These results are expected to have an important impact on ex vivo expansion of HPSCs and eventually on the design of an efficient clinicalscale expansion system. Furthermore, investigation of long-term maintenance of the HSPC phenotype, xeno-transplantation and secreted factor analyses should be performed.

This work was supported by the National Research Foundation of Korea Grant (NRF-2015M3A9B6073643) and by a grant of the Korea Health Technology R\&D Project through the KHIDI (HI16C0362, the Ministry of Health \& Welfare, ROK). 


\section{References}

[1] S. S. Kelly, et al., "Ex vivo expansion of cord blood," Bone Marrow Transplant, vol. 44, no. 10 pp. 673-681, 2009.

[2] A. Mendelson and P. S. Frenette, "Hematopoietic stem cell niche maintenance during homeostasis and regeneration," Nat. Med., vol. 20, no. 8, pp. 833-846, 2014. 